\title{
THE FACTORIZATION OF A LINEAR CONJUGATE SYMMETRIC INVOLUTION IN HILBERT SPACE
}

\author{
JAMES W. MOELLER
}

\begin{abstract}
Let $X$ be a closed linear transformation whose domain is dense in the complex separable Hilbert space $H$ and whose adjoint is denoted by $X^{*}$. The operator $X$ is said to be conjugate symmetric if $\Gamma(X) \subset \Gamma\left(Q X^{*} Q\right)$, where $\Gamma(X)$ represents the graph of $X$ in $H \oplus H$ and $Q$ is a conjugation on $H$. The main theorem in this note states that a conjugate symmetric linear involution $X$ satisfies the operator equation $X=Q X^{*} Q$.
\end{abstract}

1. Introduction. An involution is a transformation of a set into itself whose square is the identity. In this note we shall study linear involutions defined on a dense subset of a complex, infinite dimensional Hilbert space. If the involution is closed and has a polar decomposition of the form $X=U|X|$, then $U$ itself is an involution. This result is exploited to show that any conjugate symmetric involution must be conjugate selfadjoint.

In what follows $H$ will represent a complex Hilbert space having a countably infinite basis with $(f, g)$ being the inner product of two vectors in $H$. A manifold is a subset which is closed under vector addition and under multiplication by complex numbers. A subspace is a manifold which is closed in the norm topology induced by the inner product. The closure of a set $F$ in the norm topology will be denoted by clos $F$, and $F^{\perp}=\{g \mid(g, f)=0, f \in F\}$. The Hilbert space $H \oplus H$ consists of all vectors $f \oplus g$ having inner product

$$
\left(f_{1} \oplus g_{1}, f_{2} \oplus g_{2}\right)=\left(f_{1}, f_{2}\right)+\left(g_{1}, g_{2}\right) \text {. }
$$

If $X$ is a linear transformation defined on a manifold $D \subset H$, its graph

$$
\Gamma(X)=\{f \oplus X f \mid f \in D\}
$$

is another manifold in $H \oplus H$. If this manifold is a subspace, then $X$ is called a closed operator. If clos $D=H$, then $X$ is said to be densely defined. When $X$ is densely defined, it has a unique adjoint $X^{*}$ determined by the condition $(X f, g)=$ $\left(f, X^{*} g\right.$ ) which subsists for all $f$ in Dom $X$. The domain of $X^{*}$ is dense in $H$ if and only if $X$ has a closed linear extension. If $\Gamma(X) \subset \Gamma(Y)$, then $\Gamma(Y)^{\perp} \subset \Gamma(X)^{\perp}$, and this implies $\Gamma\left(Y^{*}\right) \subset \Gamma\left(X^{*}\right)$. It is not difficult to establish the inclusion $\Gamma\left(Y^{*} X^{*}\right)$ $\subset \Gamma\left((X Y)^{*}\right)$ whenever $X, Y$, and $X Y$ are all densely defined. Moreover, $(X Y)^{*}=$ $Y^{*} X^{*}$ when $X$ is a bounded linear transformation [7, p. 301]. A densely defined

Received by the editors July 11, 1984.

1980 Mathematics Subject Classification. Primary 47A05: Secondary 46 J99.

Kel nords and phrases. Hilbert space, closed linear transformation, conjugation operator. 
transformation $X$ is said to be symmetric if $\Gamma(X) \subset \Gamma\left(X^{*}\right)$. It is called selfadjoint if $X=X^{*}$. The crucial idea of representing a linear transformation through its associated graph subspace first originated in the work of J. von Neumann [5].

The next theorem plays a fundamental role in our analysis of closed involutions. Different proofs are presented in [1 and 3].

Polar Decomposition Theorem. Let $X$ be a closed linear operator whose domain is dense in $H$. Then there is a positive selfadjoint operator $|X|$ whose domain coincides with that of $X$, and a partial isometry $U$ with initial space $(\operatorname{Ker} X)^{\perp}$ and final space $\operatorname{Clos}(\operatorname{Ran} X)$ such that $X=U|X|$. This factorization is unique under the additional assumption that $\operatorname{Ker}|X|=\operatorname{Ker} X$.

2. Involutions. It is self-evident from the definition that an involution on a set $S$ is a one-to-one transformation that maps $S$ onto itself and satisfies the relation $X=X^{-1}$. If we define $V(f \oplus g)=g \oplus f$, then $X$ is an involution if and only if

$$
V(\Gamma(X)) \subset \Gamma(X) .
$$

This invariance principle leads to a simple characterization of closed, densely defined linear involutions.

LEMma 1. If $X$ is a closed linear involution whose domain $D$ is dense in $H$, there are subspaces $H^{+}$and $H^{-}$such that $H^{+} \cap H^{-}=\{0\}$ and $D=H^{+}+H^{-}$. The involution $X$ is defined by $X\left(h^{+}+h^{-}\right)=h^{+}-h^{-}$for all $h^{+}$in $\mathrm{H}^{+}$and $h^{-}$in $\mathrm{H}^{-}$. Conversely, with every pair of subspaces $H^{+}$and $\mathrm{H}^{-}$such that $\mathrm{H}^{+} \cap \mathrm{H}^{-}=\{0\}$ and $\operatorname{Clos}\left(\mathrm{H}^{+}+\mathrm{H}^{-}\right)=\mathrm{H}$, there is associated a unique densely defined closed linear involution satisfying $X\left(h^{+}+h^{-}\right)=h^{+}-h^{-}$on $D=H^{+}+H^{-}$.

Proof. Let $X$ be a closed, densely defined linear involution whose domain is $D$. Since $V$ is a unitary involution, the restriction of $V$ to the subspace $\Gamma(X)$ is a bounded selfadjoint operator whose spectrum is contained in the two point set $\{-1,1\}$. If $J$ denotes the restriction of $V$ to the subspace $\Gamma(X)$, then the subspaces $H_{0}^{+}=\{f \oplus g \mid J(f \oplus g)=f \oplus g\}$ and $H_{0}^{-}=\{f \oplus g \mid J(f \oplus g)=-(f \oplus g)\}$ are mutually orthogonal. The spectral theorem for selfadjoint operators further asserts that $\Gamma(X)=H_{0}^{+} \oplus H_{0}^{-}$. If we define $H^{+}=P\left(H_{0}^{+}\right)$and $H^{-}=P\left(H_{0}^{-}\right)$, where $P(f \oplus g)=f$, it is easy to see that $X\left(h^{+}+h^{-}\right)=h^{+}-h^{-}$on $D=H^{+}+H^{-}$. Clearly, both $H^{+}$and $H^{-}$are subspaces.

Now suppose that $H^{+}$and $H^{-}$are two subspaces such that $H^{+} \cap H^{-}=\{0\}$ and $\operatorname{Clos}\left(H^{+}+H^{-}\right)=H$. Define the transformation $X$ by the equation

$$
X\left(h^{+}+h^{-}\right)=h^{+}-h^{-} .
$$

If $h_{n}^{+}+h_{n}^{-} \rightarrow f$ and $h_{n}^{+}-h_{n}^{-} \rightarrow g$, then the vector $(f+g) / 2$ belongs to $H^{+}$and the vector $(f-g) / 2$ belongs to $H^{-}$. Hence $X(f)=g$, and we conclude that $X$ is a closed, densely defined involution uniquely associated with the subspaces $H^{+}$and $H^{-}$.

THEOREM 1. Let $X$ be a closed, densely defined linear involution whose polar representation has the form $X=U|X|$. Then $U$ is a unitary involution. 
Proof. Since $X$ is an involution, $\operatorname{Ker}|X|=\{0\}$ and $U$ is a unitary operator on $H$. From the identity $X=X^{-1}$ we immediately obtain $U|X|=|X|^{-1} U^{*}$. Therefore $U|X| U=|X|^{-1}$ and, after taking adjoints, we get $U^{*}|X| U^{*}=|X|^{-1}$. Hence $U^{2}|X| U^{2}=|X|$, from which it follows that $U^{2}|X|=|X| U^{* 2}$ because $U$ is a unitary operator. This last equation immediately implies $U^{2}|X|$ is selfadjoint. Now let $H^{+}=\left\{h^{+} \mid X\left(h^{+}\right)=h^{+}\right\}$and $H^{-}=\left\{h^{-} \mid X\left(h^{-}\right)=-h^{-}\right\}$. Then $|X| h^{+}=U^{*} h^{+},|X| h^{-}$ $=-U^{*} h^{-}$, and

$$
\begin{aligned}
\left(U^{2}|X|\left(h^{+}+h^{-}\right), h^{+}+h^{-}\right) & =\left(|X|\left(h^{+}+h^{-}\right), U^{* 2}\left(h^{+}+h^{-}\right)\right) \\
& =\left(U^{*}\left(h^{+}-h^{-}\right), U^{* 2}\left(h^{+}+h^{-}\right)\right) \\
& =\left(h^{+}-h^{-},|X|\left(h^{+}-h^{-}\right)\right) .
\end{aligned}
$$

Thus $\left(U^{2}|X|\left(h^{+}+h^{-}\right), h^{+}+h^{-}\right) \geqslant 0$ for all $h^{+}$in $H^{+}$and $h^{-}$in $H^{-}$. Since Dom $X=$ $H^{+}+H^{-}$by virtue of Lemma $1, U^{2}|X|$ is a positive selfadjoint operator on Dom $X$. The uniqueness of the polar decomposition implies $U^{2}=I$.

3. Conjugate symmetric involutions. A transformation $Q$ on $H$ that satisfies $Q(\alpha f+\beta g)=\alpha^{*} Q(f)+\beta^{*} Q(g)$ is called a conjugation if $Q^{2}=I$ and $(Q f, Q g)=$ $(g, f)$ for every $f$ and $g$ in $H$. We remark in passing that theoretical physicists have found conjugation operators to be useful tools for studying time reversal symmetry in quantum mechanics [2, p. 187]. If there is a conjugation $Q$ such that $X=Q X^{*} Q$, we shall call $X$ conjugate selfadjoint. If $\Gamma(X) \subset \Gamma\left(Q X^{*} Q\right)$, then $X$ is said to be conjugate symmetric. Commutativity properties of bounded conjugate selfadjoint operators are treated in [4], but little appears to be known about their general structure.

LEMMA 2. Let $X$ be a closed, densely defined linear involution having the property that $\Gamma(X) \subset \Gamma\left(Q X^{*} Q\right)$. If $X=U|X|$ is the polar representation for $X$, then $U Q(\operatorname{Dom} X) \subset \operatorname{Dom} X$ and $\operatorname{Dom}|X| \subset \operatorname{Dom} Q|X|^{-1} Q$.

Proof. An application of Theorem 1 immediately yields the identity $U X U=X^{*}$. Therefore $\Gamma(X) \subset \Gamma(Q U X U Q)$, and it follows that $U Q(\operatorname{Dom} X) \subset \operatorname{Dom} X$. Since Dom $X=\operatorname{Dom}|X|$ and $Q|X|^{-1} Q=Q U|X| U Q$, we further have $\operatorname{Dom}|X| \subset$ $\operatorname{Dom}\left(Q|X|^{-1} Q\right)$.

LEMMA 3. Let $X$ denote the conjugate symmetric involution introduced in the previous lemma, and let $V=Q|X|^{-1} Q|X|^{-1}$. Then $V$ is a densely defined linear transformation having the property that $\operatorname{Dom} V=\operatorname{Dom} X^{*}$ and $\Gamma(V) \subset \Gamma(Q U Q U)$.

Proof. Substituting $X^{*}=U|X|^{-1}$ in the defining equation for $V$, we get $V=$ $Q U X^{*} Q U X^{*}$. This immediately implies that $\operatorname{Dom} V \subset \operatorname{Dom} X^{*}$. But $\Gamma(X) \subset$ $\Gamma\left(Q X^{*} Q\right)$ by hypothesis, so we have $\Gamma\left(Q U Q U X^{* 2}\right)=\Gamma\left(Q U Q X U X^{*}\right) \subset \Gamma(V)$. Since $X^{* 2}$ is the identity on $\operatorname{Dom} X^{*}$, it follows that $\operatorname{Dom} V=\operatorname{Dom} X^{*}$ and $\Gamma(V) \subset \Gamma(Q U Q U)$ because $Q U Q U$ is a unitary transformation defined on all of $H$.

TheOrem 2. If $X$ is a closed, densely defined linear involution which is conjugate symmetric, then $X$ is conjugate selfadjoint. 
Proof. Let $K$ be the linear transformation given by $K=2|X|+Q|X|^{-1} Q$. According to Lemma 2, Dom $K=\operatorname{Dom}|X|$, and we can thus write $K=$ $\left(2+Q|X|^{-1} Q|X|^{-1}\right)|X|$. An application of Lemma 3 now gives $K=R|X|$, where $R=2+Q U Q U$. Since $R$ is a bounded linear transformation on $H$ whose spectrum $\sigma(R)$ has the property that $\sigma(R) \subset\{z \mid \operatorname{Re} z \geqslant 1\}$, it follows that $K$ is a closed linear operator with Dom $K=\operatorname{Dom}|X|$. Moreover, since $\Gamma\left(Y^{*}+Z^{*}\right) \subset \Gamma\left((Y+Z)^{*}\right)$ for densely defined linear transformations [7, p. 300], we obtain $\Gamma(K) \subset \Gamma\left(K^{*}\right)$. Hence $\Gamma(R|X|) \subset \Gamma\left(|X| R^{*}\right)$ and it follows that $\left(R^{*} \oplus R\right) \Gamma(|X|)=\Gamma\left(R|X| R^{*-1}\right) \subset \Gamma(|X|)$. Since $\sigma\left(R^{*} \oplus R\right)=\sigma\left(R^{*}\right) \cup \sigma(R) \subset\{z \mid \operatorname{Re} z \geqslant 1\}$, any invariant subspace for $R^{*}$ $\oplus R$ is also an invariant subspace for $\left(R^{*} \oplus R\right)^{-1}[6$, p. 33]. Consequently $\left(R^{*} \oplus R\right) \Gamma(|X|)=\Gamma(|X|)$, and we conclude that $Q U Q U|X|=|X| U Q U Q$. This last equation implies

$$
U Q U Q(\operatorname{Dom} X)=\operatorname{Dom} X=Q U Q U(\operatorname{Dom} X),
$$

so we finally have $X=Q X^{*} Q$.

COROllary. If $X$ is a conjugate symmetric linear involution and $Y$ is a closed densely defined linear operator having the property that $\Gamma(Y) \subset \Gamma(X)$, then $Y=X$.

Proof. From the hypotheses we at once find

$$
\Gamma(Y) \subset \Gamma(X) \subset \Gamma\left(Q X^{*} Q\right) \subset \Gamma\left(Q Y^{*} Q\right)
$$

Consequently $Q Y^{*} Q=Y$ by virtue of Theorem 2, and this implies $X=Y$.

\section{REFERENCES}

1. N. Dunford and J. T. Schwartz, Linear operators. Part II: Spectral theory, Wiley, New York, 1963.

2. L. M. Falicov, Group theory and its physical applications, Univ. of Chicago Press, Chicago, Ill., 1966.

3. T. Kato, Perturbation theory for linear operators, Springer-Verlag, New York, 1966.

4. J. Moeller, $A$ double commutant theorem for conjugate selfadjoint operators, Proc. Amer. Math. Soc. 83 (1981), 506-508.

5. J. von Neumann, Uber adjungierte Funktionaloperatoren, Ann. of Math. 33 (1932), 294-310.

6. H. Radjavi and P. Rosenthal, Invariant subspaces, Springer-Verlag, New York, 1973.

7. F. Riesz and B. Sz.-Nagy, Functional analysis, Ungar, New York, 1955.

Di:partment of Mathematics, University of Illinois at Chicago, Chicago, Illinois 60680 